\title{
Analysis of Simulated Output Characteristics of Gas Sensor Based on Graphene Nanoribbon
}

\author{
A. Mahmoudi, ${ }^{1}$ M. Troudi, ${ }^{2}$ Y. Bergaoui, ${ }^{2}$ P. Bondavalli, ${ }^{3}$ and N. Sghaier ${ }^{1,2}$ \\ ${ }^{1}$ Laboratory of Microelectronics and Instrumentation (LR13ES12), Faculty of Sciences of Monastir, Environnement Street, \\ 5019 Monastir, Tunisia \\ ${ }^{2}$ Team of Electronic Devices (UR11ES89), Institut Preparatoire aux Etudes d'Ingénieurs de Nabeul (IPEIN), University of Carthage, \\ Merazka, 8000 Nabeul, Tunisia \\ ${ }^{3}$ Nanocarb Laboratory, Thales Research and Technology, 91767 Palaiseau, France
}

Correspondence should be addressed to N. Sghaier; nabil.sghaier@ipein.rnu.tn

Received 27 February 2016; Revised 16 May 2016; Accepted 23 June 2016

Academic Editor: Elisabetta Comini

Copyright (C) 2016 A. Mahmoudi et al. This is an open access article distributed under the Creative Commons Attribution License, which permits unrestricted use, distribution, and reproduction in any medium, provided the original work is properly cited.

\begin{abstract}
This work presents simulated output characteristics of gas sensor transistors based on graphene nanoribbon (GNRFET). The device studied in this work is a new generation of gas sensing devices, which are easy to use, ultracompact, ultrasensitive, and highly selective. We will explain how the exposure to the gas changes the conductivity of graphene nanoribbon. The equations of the GNRFET gas sensor model include the Poisson equation in the weak nonlocality approximation with proposed sensing parameters. As we have developed this model as a platform for a gas detection sensor, we will analyze the current-voltage characteristics after exposure of the GNRFET nanosensor device to $\mathrm{NH}_{3}$ gas. A sensitivity of nearly $2.7 \%$ was indicated in our sensor device after exposure of $1 \mathrm{ppm}$ of $\mathrm{NH}_{3}$. The given results make GNRFET the right candidate for use in gas sensing/measuring appliances. Thus, we will investigate the effect of the channel length on the ON- and OFF-current.
\end{abstract}

\section{Introduction}

The gas detection is very important in both research and commercial applicability. There is an increasing need for a higher sensitivity and selectivity as well as for a faster response time. The most significant feature of a material in gas sensing applications is its elevated surface-to-volume ratio. For this motive, new materials are revealed in the construction of innovative sensors with an elevated surface-tovolume ratio. This grants a considerable active surface area for the gas molecule interactions. The different patterns of these nanostructures are graphene and carbon nanotubes (CNT) $[1,2]$, highly enhancing the absorption of gases and eventually resulting in a better sensitive sensor performance [3]. CNT have been presented as an ideal material for developing sensor technology because of their one-dimensional (1D) structures with high surface-to-volume ratio that enables them to integrate as gas sensing. Despite their promises, CNT still face considerable challenges due to the existing growth methods which do not allow controlling the structural uniformity such as diameter, number of graphene walls, and electrical properties by separating metallic and semiconducting CNT and so CNT involved structural heterogeneity [4]. However, graphene also presented the possibility of high sensitivity towards gas sensing, in addition to several advantages over CNT-based sensors. Graphene is shown to exhibit inherently low electrical noise at ambient temperature and high electron mobility, which enhance its sensitivity compared to CNT. Also, compared to CNT, graphene is relatively easier for electrical manipulation [4].

Graphene is defined as a single stratum of carbon atoms that are arranged in a two-dimensional honeycomb lattice. Since its inception in 2004, this material attracted enormous attention, which led to an intensive research activity [5]. As a result of its zero band gap energy, the electron mobility in graphene is 100 times quicker than that in silicon and makes it a perfect two-dimensional (2D) semiconductor [6-8]. When graphene is cut into thin graphene strips (nanoribbons) a sizeable band gap opens. In these nanoribbons, the confinement of transporters leads to a quasi-one-dimensional 
(1D) system creating an energy gap. Comparable to the CNT, this energy gap relies on the width and atom direction of the graphene nanoribbon (GNR) [9]. The calculation of density functional theory (DFT) demonstrates that armchair nanoribbons are semiconductors. The latter energy gap is inversely proportional to the GNR width [10]. Specifically, when we precisely determine the nanoribbons width, we can put together the graphene structures with a fairly wide band gap, keeping a rather high electron (hole) mobility [11]. Experiments verified that energy gaps augment with decreasing GNR width [12]. It has been stated that armchair nanoribbons of $2.5 \mathrm{~nm}$ width showed an energy gap getting up to $0.5 \mathrm{EV}$.

Furthermore, CNT unique electronic characteristics like tunable conductance, ballistic transport, and highly charged mobility [13] create an area of strong interest for sensing application in nanotechnology [14]. In this context, Schedin et al. reported the potential application of graphene in sensing applications in 2007 [15]. More specifically, GNR has the ability to detect adsorbed molecules onto the surface (e.g., $\mathrm{NH}_{3}, \mathrm{NO}_{2}, \mathrm{CO}, \mathrm{H}_{2} \mathrm{~S}$, and $\mathrm{NO}_{2}$ ). To date, highly sensitive and selective sensors as well as quick response times are preferred. Graphene nanoribbon was the most interesting kind of nanomaterials. They contain unique properties, which include powerful electrical and thermal conductivity and high surface-to-volume ratios. The gas interaction with graphene nanoribbon is a very interesting area in terms of basic research and in terms of possible applications as gas detector. Appropriate configuration for graphene nanoribbon use in measuring devices is where the GNR is set as a channel between two electrodes of a field effect transistor. After exposure to the gas, the detection signals of our sensor device are obtained through the changes in the $I-V$ curves of graphene field effect transistor. That makes GNRFET a favorable device sensor and shows a high sensitivity towards any alterations in the chemical environment [16].

In the present research we will propose a basic model of how GNRs can be used in gas detection applications. The recommendations from the mathematical model will highlight the relevant properties of graphene in the context of gas sensing, which will elucidate the fundamental working principle of chemical sensors. We have employed the field effect transistor (FET) as a basic structure of our gas sensor model.

\section{Graphene Nanoribbon-Based Sensors}

The model's focus was supposed to merge the merits and advantages of graphene nanoribbon which has broad specific surface area $\left(2630 \mathrm{~m}^{2} \mathrm{~g}^{-1}\right)$ [4] providing great sensing area per unit volume to adsorb chemical species and high electron mobility at ambient temperature.

The structure of the proposed analytical device using graphene nanoribbon as a channel for gas sensor is the same as the metal-oxide semiconductor field effect transistors (MOSFETs) structure [17-19]. The GNRFET we are considering is based on a graphene layer which composes a dense array of parallel nanoribbons. The nanoribbon used as a transistor channel is connected between the source and drain

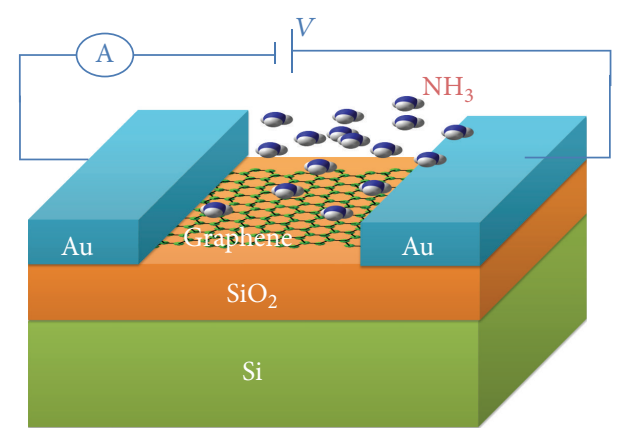

FIGURE 1: GNRFET-based gas sensor.

electrodes. We have used a dielectric layer which separates the gate from the channel. Here, $\mathrm{SiO}_{2} / \mathrm{Si}$ substrate is used as a bottom gate (Figure 1).

The nanoribbon surface could interact with gas molecules, changing the GNRFET conductivity and so its transfer characteristics due to the variability of the source-drain current which is a measurable parameter [4]. This effect can be explained by a kind of "molecular gating" of the gas molecules on the GNRFET channel.

Recently, many theoretical studies on the molecular gas adsorption on the GNR have been carried out. Those studies indicate that the adsorption of $\mathrm{CO}_{2}$ and $\mathrm{O}_{2}$ molecules results in a $p$-type semiconductor while the $\mathrm{NH}_{3}$ gas adsorption generates $n$-type features [20]. $\mathrm{NH}_{3}$ and $\mathrm{CO}$ gases serve as donors, while $\mathrm{H}_{2} \mathrm{O}$ and $\mathrm{NO}_{2}$ act as acceptors [21]. In our case, we will focus on the graphene nanoribbon conductance exposed to the $\mathrm{NH}_{3}$ molecules. The $\mathrm{NH}_{3}$ gas molecule adsorption will donate electrons to the GNR surface which changing the GNR conductance and thus the $I-V$ curves of our GNRFET-based gas sensor. With the suggested sensing parameters, we consider this phenomenon as the detection mechanism. We have proposed the transfer characteristics of the GNR-based detector as a principle by which to detect the effects of gas adsorption.

\section{GNRFET-Based Gas Sensor Model}

In our model we consider that the GNRFET operates with the condition that the channel's electron gas is nondegenerate. Thus, the distribution functions of the electron and the hole in the subbands near the source and drain electrodes are shown in the following [22]:

$$
\varepsilon_{p, 1}^{-1}= \pm v \sqrt{p^{2}+\left(\frac{\pi \hbar}{d}\right)^{2}} .
$$

Here $v=108 \mathrm{~cm} / \mathrm{s}$ is the characteristic charge velocity, the $( \pm)$ signs refer to the electron (upper sign) and hole (lower sign) spectra, $d=2.06 \mathrm{~nm}$ is the nanoribbon width, $\hbar$ is the reduced Planck constant, and $p$ is the momentum along the nanoribbon.

The proposed model is based on the correlation between current, gas concentration, and temperature as follows:

$$
I_{\text {gas-GNR }}=I_{w g}+I_{g T}+I_{g F} \text {. }
$$


When the proposed device is exposed to the gases at different temperatures, we can define three current parameters of the GNRFET-based gas sensor: $I_{w g}, I_{g T}$, and $I_{g F}$, where $I_{w g}$ is the current of the device without gas exposure which takes account of the different geometric parameters of the device; $I_{g T}$ represents changes in the current which depends on the temperature $(T)$; and $I_{g F}$ modulate the dependence of the current on different values of gas concentration at a stable temperature. The conductance of the gas sensor changes for different values of $\mathrm{NH}_{3}$ gas concentrations and for various temperatures $[14,17]$. In what follows, we will define each parameter.

3.1. The Current without Gas Exposure. The nanoribbon sections adjacent to the source and drain contacts are serving as conducting pads and are highly conducting. Thus, these sections are equipotential and equal to $\varphi=0$ and $\varphi=V_{d}$, respectively. The active region of the GNRFET which replaces the top gate is defined as follows: $L_{g} \leq x \leq L_{g} / 2, W_{b} \leq z \leq 0$, where $L_{g}$ is the length of the active region (top gate) and $W_{b}$ is the thickness of the layer between the graphene and the back gate. Potential distribution along the channel can be found using the two-dimensional Poisson equation for the electric potential $\psi=\psi(x, z)$ for the active region under consideration in the weak nonlocality estimation $\varphi=\varphi(x)=$ $\psi(x, 0)[23,24]$ as follows [22]:

$$
\frac{W_{b}}{3} \frac{\partial^{2} \varphi}{\partial x^{2}}-\frac{\varphi-V_{b}}{W_{b}}-\frac{\varphi-V_{g}}{W_{g}}=\frac{4 \pi e}{æ}\left(\Sigma_{-}-\Sigma_{+}\right),
$$

where, $\Sigma_{-}$and $\Sigma_{+}$are the electron and hole densities in the channel, $e$ is the electron charge, and $æ$ is the material dielectric constant separating the channel from the back gate. Equation (2) controls the electric potential. The approximation under consideration makes the study of nonuniform potential distributions in the GNRFET channel and the shortgate effects analytically possible.

The Fermi energies, the electric potential, and the electron densities in the source and drain regions are related to each other as

$$
\begin{aligned}
& \Sigma_{\mp}^{s}=\frac{2 \sqrt{2 \Delta K_{B} T}}{\sqrt{\pi} \hbar d v} \exp \left( \pm \frac{\varepsilon_{f}^{s}+e \varphi}{K_{B} T}-\frac{\Delta}{2} K_{B} T\right), \\
& \Sigma_{\mp}^{d}=\frac{2 \sqrt{2 \Delta K_{B} T}}{\sqrt{\pi} \hbar d v} \exp \left( \pm \frac{\varepsilon_{f}^{d}+e\left(\varphi-V_{d}\right)}{K_{B} T}-\frac{\Delta}{2} K_{B} T\right),
\end{aligned}
$$

where $\Delta=4 \pi \hbar / d$ is the value of the energy band gap.

If we consider that the current is determined by the electrons overcoming the potential barrier between GNR and electrodes, we can use the current formula as the follows (per unit length) [22]:

$$
I_{w g}=\frac{2 e}{\pi \hbar d}\left(\int v_{p} f_{p}^{s} d p-\int v_{p} f_{p}^{d} d p\right) .
$$

Here

$$
v_{p}=\frac{d \varepsilon_{p, 0}^{+}}{d P}=v^{2} \frac{P}{\sqrt{v^{2} P^{2}+\Delta^{2} / 4}}
$$

is the velocity of the electron with momentum $p$ of the

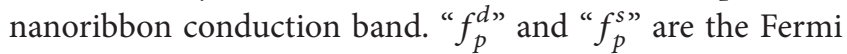
energy determined from the middle of the energy band gap. Integrating in (5), we arrive at [22]

$$
\propto \begin{cases}I_{w g}\left[\frac{e V_{g}}{K_{B} T}\left(1-\frac{1}{\cosh \left(L_{g} / 2 \Lambda\right)}\right)\right] ; & \text { at }\left|V_{g}\right| \leq \frac{V_{b}}{W_{b}}, \\ V_{b} \exp \left[\frac{e V_{g}}{K_{B} T}\left(1-\frac{1}{\cosh \left(L_{g} / 2 \Lambda\right)}\right)\right] ; & \text { at }\left|V_{g}\right|>\frac{V_{b}}{W_{b}} .\end{cases}
$$

Here $\Lambda=\sqrt{\left(W_{b} / 3\right)}$ is the effective screening length.

3.2. The Current with Gas Exposure. We can associate $I_{g T}$ and $I_{g F}$ as one parameter depending on each other as [14]

$$
\begin{aligned}
I_{g} & =I_{g T}+I_{g F} \\
& =\frac{2 e}{\pi \hbar d}\left(\int \frac{x^{-1 / 2}}{1+e^{x-\eta}} d x+\int \frac{x^{-1 / 2}}{1+e^{x+\eta}} d x\right),
\end{aligned}
$$

where $\eta=\left(E_{F}-E_{G}\right) / k_{B} T$ and $x=\left(E-E_{G}\right) / k_{B} T$ are the normalized Fermi energy. We can solve this equation numerically by using the partial integration method $[25,26]$. Thus, the general model for this part of the graphene-based gas sensors current can be derived similarly to that of the silicon-based model proposed by Gunlycke et al. [27]:

$$
\begin{aligned}
& I_{g} \\
& \qquad \begin{array}{l}
\pi \hbar d \\
\quad * V_{g} .
\end{array}
\end{aligned}
$$

Here $\xi_{-1 / 2}$ is the Fermi-Dirac integral of order $(-1 / 2)$ which plays a significant role in the modeling of semiconductor's behavior. $E_{G}$ strongly influences the gas sensor conductance because it depends on gas concentration and its temperature [14]. We can write it as follows:

$$
E_{G}=\delta T+\lambda F,
$$

where $\delta$ is the parameter that controls temperature and $\lambda$ is the parameter which monitors the gas concentration. These parameters are obtained by iteration method [14]. Equations (9) and (10) are used to obtain the gas sensor current model as

$$
\begin{aligned}
I_{g} & =\frac{2 e}{\pi \hbar d}\left(\xi_{-1 / 2}\left(\frac{\left(E_{F}-(\delta T+\lambda F)\right)}{k_{B} T}\right)\right. \\
& \left.+\xi_{-1 / 2}\left(\frac{\left(\delta T+\lambda F-E_{F}\right)}{k_{B} T}\right)\right) * V_{g} .
\end{aligned}
$$

Finally, summing (7) and (11) we obtain the general source-drain current as a function of the top gate voltage, 


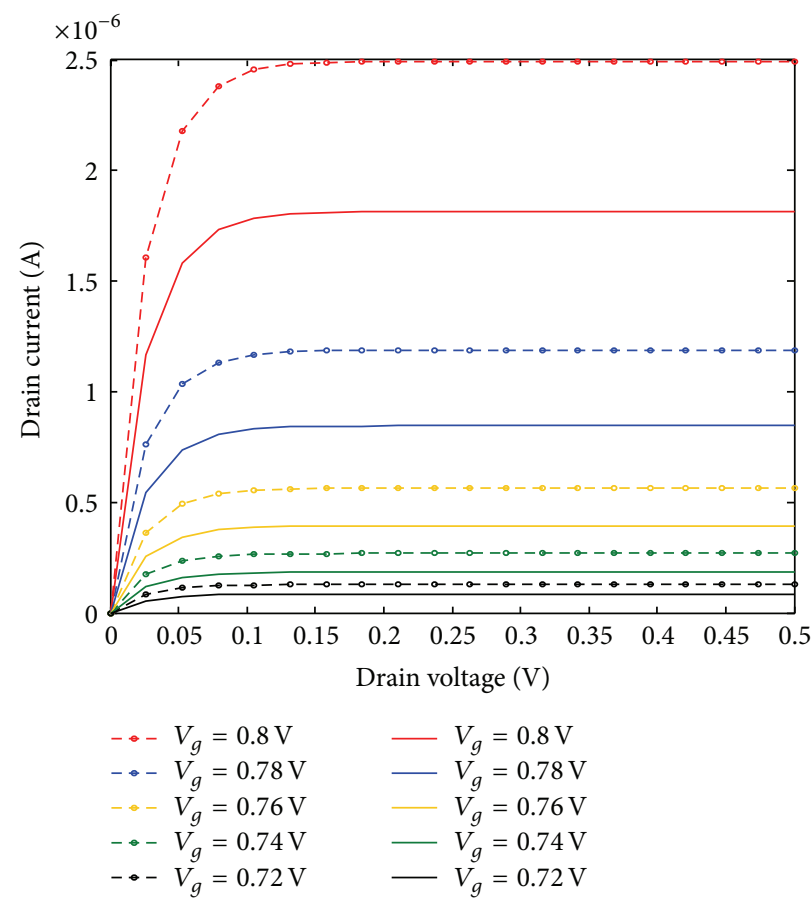

FIgURE 2: The $I-V$ curves before (solid line) and after (dashed line) $\mathrm{NH}_{3}$ exposure at $F=300 \mathrm{ppm}$.

the graphene length, the gas concentration, and temperature by the following relations:

$$
\begin{aligned}
& I_{\text {gas-GNR }}
\end{aligned}
$$

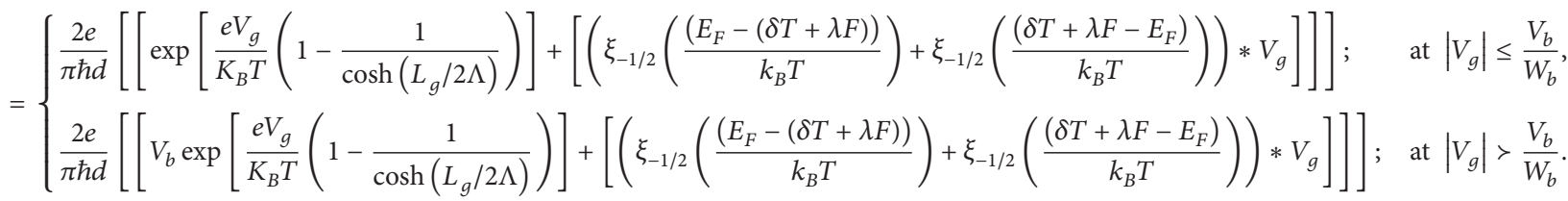

Using this equation, we can evaluate the performance of graphene-based gas sensor FET devices based on $(I-V)$ curves. We can control the drain-source current by monitoring the top gate voltage which changes as the channel is exposed to gas. Our model works in ohmic and saturation regions, quite similar to MOSFETs.

\section{Results and Discussion}

4.1. GNR-Based Gas Sensor Characteristics at Room Temperature. The sensing measurements of graphene-FETs were carried out using $\mathrm{NH}_{3}$ gases, which behave as electron donor [28]. In order to evaluate the sensitivity of our device, we first have studied the current-voltage characteristics profile of graphene gas sensor transistors at various gate biases (varying from $0.72 \mathrm{~V}$ to $0.8 \mathrm{~V}$ ) before gas exposure (solid lines) and after exposure to $\mathrm{NH}_{3}$ (dashed lines) at a concentration of $300 \mathrm{ppm}$ at room temperature.

Figure 2 shows that the $I-V$ characteristics of the GNRFET have increased after exposure to $\mathrm{NH}_{3}$. The gas molecules are then adsorbed on the graphene and modify its conductivity. This behavior is expected, because $\mathrm{NH}_{3}$ is an electron donor, which increases the density of electrons in GNRFET following adsorption, and increases in the conductivity of $n$ type graphene.

In Figure 3(a), we indicate the $I-V$ curves after $\mathrm{NH}_{3}$ exposure at $300 \mathrm{~K}$ and at different gas concentration values. It is evident that when we increase the gas concentration the conductivity increases as well. We can observed the sensitivity from the responses of our device under $350 \mathrm{ppm}, 400 \mathrm{ppm}$, $450 \mathrm{ppm}$, and $500 \mathrm{ppm} \mathrm{NH}_{3}$.

The sensitivity of GNRFET is defined as a percentage conductance change after gas exposure. It can be calculated as $100 \times\left(I_{g}-I_{w g}\right) / I_{w g}$, where $I_{w g}$ is the base current in the absence of the gas and $I_{g}$ is the current in the presence of the gas $[4,12,29]$. Here, as shown in Figure 3(b) we found that, upon exposure to $450 \mathrm{ppm} \mathrm{NH}_{3}$, the sensitivity increased by $55 \%$ at $V_{g}=0.8$ and $T=300 \mathrm{~K}$.

The behavior of the sensitivity of the sensor is practically linear. The GNR gas detectors reveal an increase of almost 


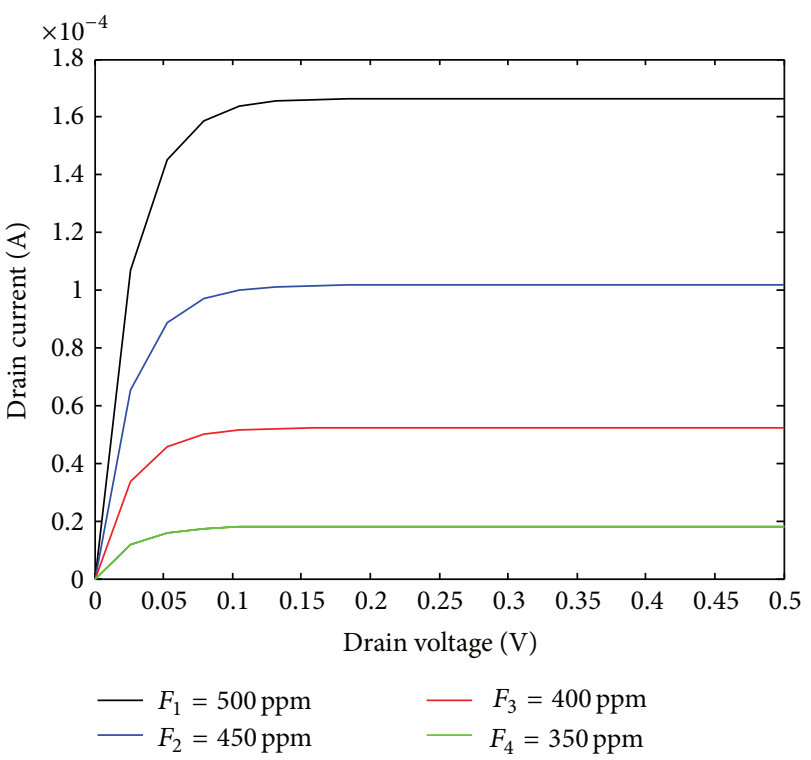

(a)

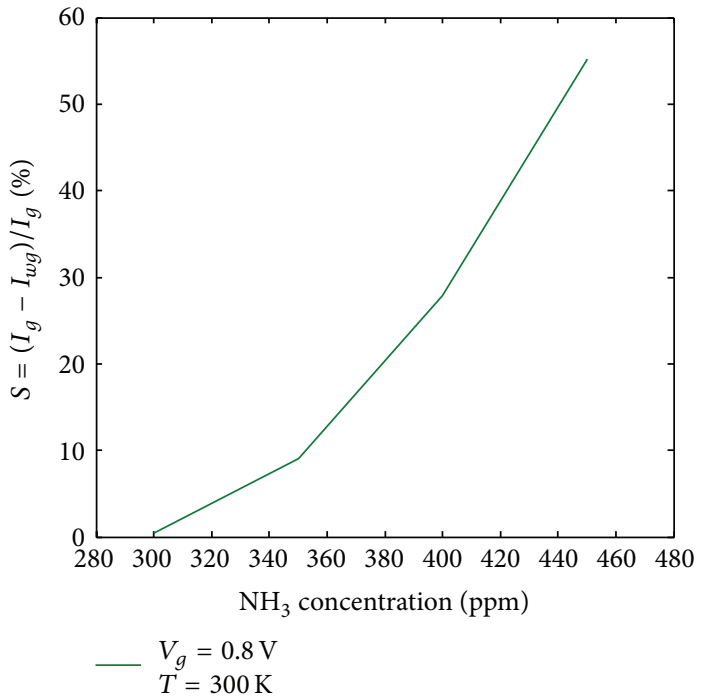

(b)

Figure 3: (a) Change in $I-V$ response of the graphene sensor at different gas concentrations. (b) Change in sensitivity with different concentrations of $\mathrm{NH}_{3}$ exposure.

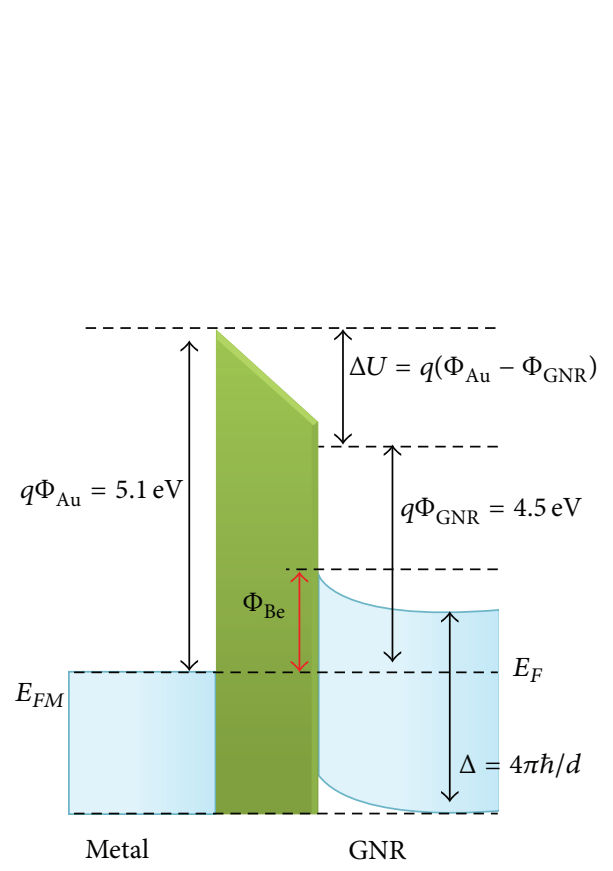

(a)

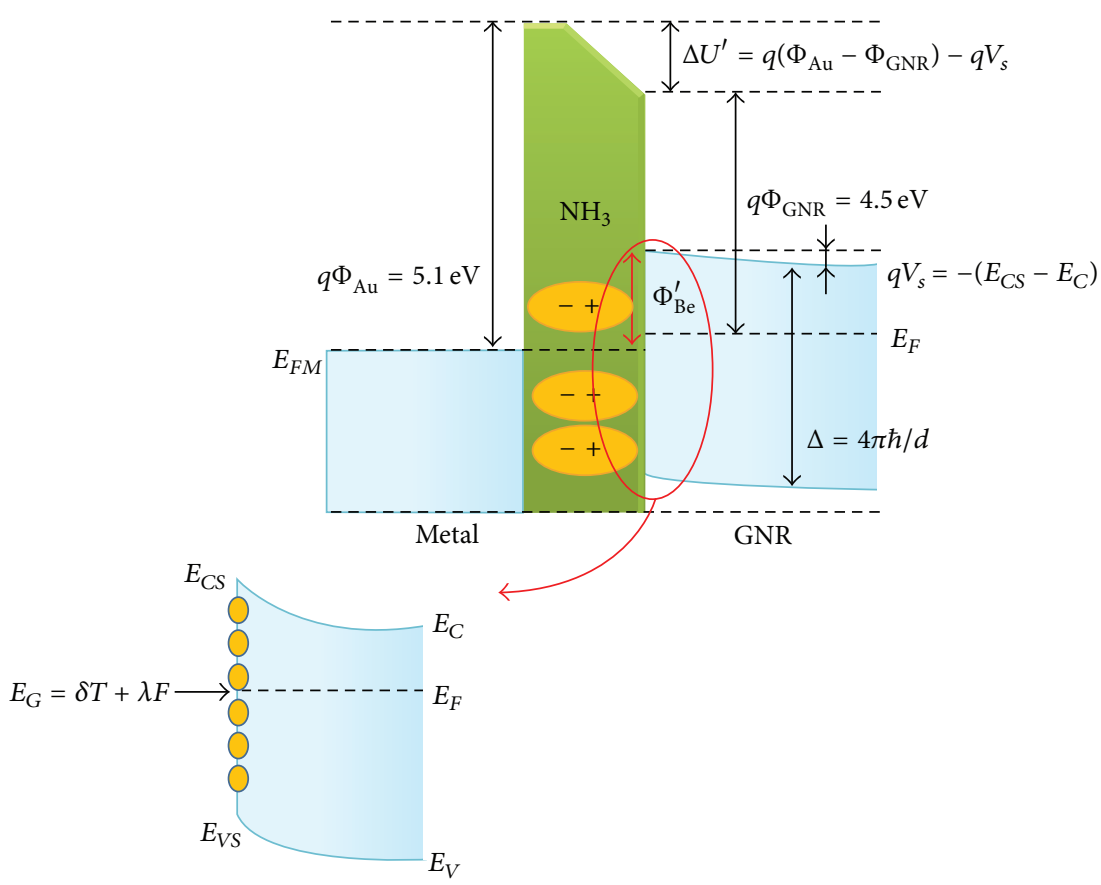

(b)

FIGURE 4: Schottky junction built up between the GNR and the electrodes: (a) before gas exposure and (b) after exposure to $\mathrm{NH}_{3}$.

$2.7 \%$ in sensitivity to exposure of $1 \mathrm{ppm}$ of $\mathrm{NH}_{3}$ concentration. This phenomenon could be explained considering that our device exploits an important transformation of the Schottky junctions built up between the graphene nanoribbon and the electrodes (Figure 4): an interfacial dipole is induced by the adsorption of the $\mathrm{NH}_{3}$ gas. This results in a change of the bending and height of the Schottky barrier at the contacts [30].
Consequently, we notice a change in the alignment of the Fermi level between the drain/source electrodes and the GNR bands. Moreover, the Schottky barrier characteristics at the junction change as well. This results in the electron injection at the barrier and the metal/GNR junction is decreased, giving rise to the current. This consideration is very important because it seems to confirm that the interaction between the gases and the metal/nanoribbon is the phenomenon 


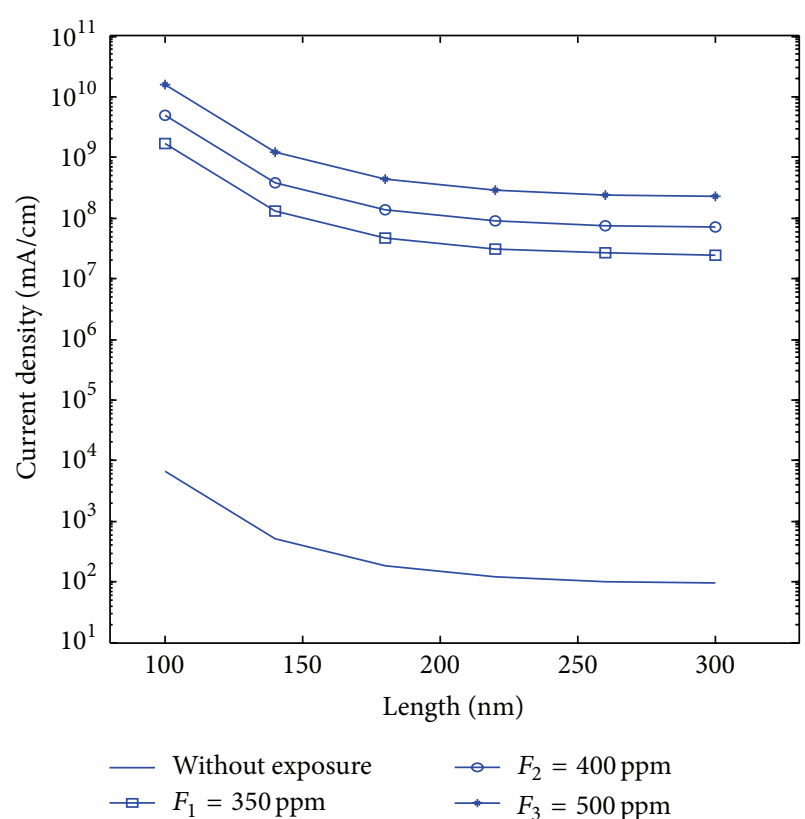

(a)

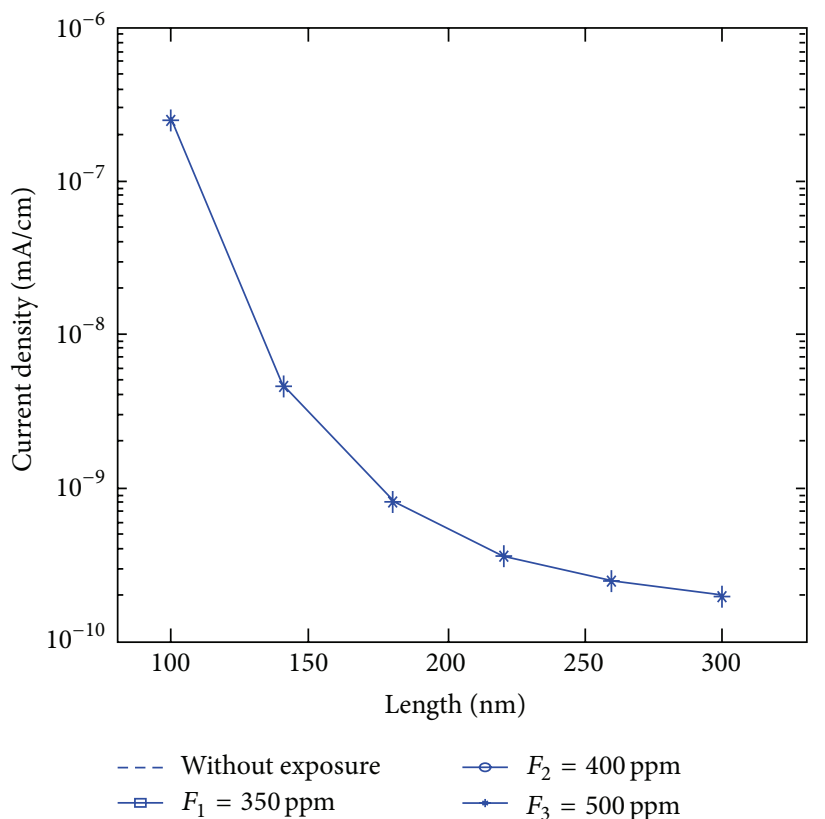

(b)

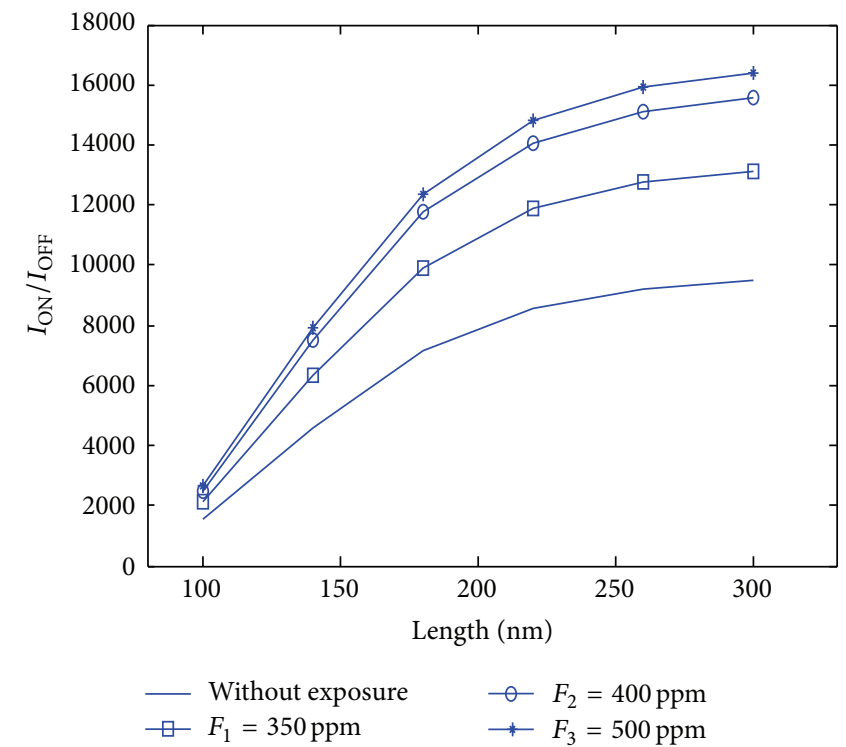

(c)

FIgURE 5: The (a) ON- and (b) OFF-current curve and (c) $I_{\mathrm{ON}} / I_{\mathrm{OFF}}$ ratio as a function of the device length before exposure (simple line) and after exposure to different gas concentration (line + symbol).

at the base of sensing for this kind of sensors. Thus, by measuring the variation of the electrical conductivity, due to the interaction with the contacts, we can identify the detected molecule and also the concentration in the air of the found chemical species.

To confirm this point of view, we have simulated the variation of the $I_{\mathrm{ON}}$ and $I_{\mathrm{OFF}}$ curves as a function of the channel length at different gas concentrations. As shown in Figures 5(a) and 5(b), there is a lateral shift of the $I_{\mathrm{ON}}$ without any shift of the OFF-current interval. Thus we can conclude that exposure to a gas does not dope the graphene nanoribbon GNRFET sensor. The doping effect would imply a change in the "OFF" range of the transistor. In reality, the gas molecule adsorption changes the Fermi level alignment between the graphene nanoribbon and the metal electrodes in the contact region.

This statement is demonstrated by the comparison between the measurements performed by changing the gas concentration exposed to the GNRFET. This consideration is in agreement with the analytical and experimental results in the instance of oxygen exposition attained by an IBM team in Yorktown Heights, led by Avouris et al. [31].

As is seen in Figure 5, the channel length effect indicated on the device characteristics at different gas concentration is 
investigated. Actually, as the device length diminishes, both the ON- and OFF-currents raise. As shown in Figure 5(a), the ON-current moves up by a factor of about 33.26, by ranging the total length of the device $L$ from 300 to $100 \mathrm{~nm}$. The OFF-current increases by a factor of $1.25 e^{-9}$ with the same channel length scaling (Figure 5(b)). As an outcome to that, the $I_{\mathrm{ON}} / I_{\mathrm{OFF}}$ ratio increases from $1.53 e^{3}$ at $L=100 \mathrm{~nm}$ to $9.48 e^{4}$ at $L=300 \mathrm{~nm}$ before gas exposure and from $2.51 e^{3}$ at $L=100 \mathrm{~nm}$ to $1.31 e^{4}$ at $L=300 \mathrm{~nm}$ after $\mathrm{NH}_{3}$ exposure at $F=350 \mathrm{ppm}$.

This result can be credited to an essential reliance of the height of the prospective barrier built up between the GNR and the drain/source metal electrodes for the electrons propagating on the molecular gate length (the nanoribbon). In the long-channel limit, the OFF-current is mostly owed to the thermionic discharge of carriers over the impending barrier, whereas in the short-channel limit the OFF-current is dominated by the tunneling of carriers through the barrier [32]. In particular, as seen from (7) and (12), in GNRFET with a long top gate, the source-drain current soaked when $V_{d}$ becomes larger that $\left(k_{B} T / e\right)$, whereas in GNRFET with $L_{g}$ comparable with $\Lambda$ the source-drain current noticeably amplifies with increasing $V_{d}$ even at rather big values of the latter.

4.2. GNR-Based Gas Sensor Characteristics at Elevated Temperature Region. As shown in Figure 6, the $I-V$ curves of the GNR-based gas sensor equivalent to temperatures of 23, 50, and $100^{\circ} \mathrm{C}$, respectively, are illustrated (line with symbols), after exposure to $300 \mathrm{ppm} \mathrm{NH}_{3}$, as well as the $I-V$ before gas exposure (solid line).

It can be seen that the drain current has increased when the temperature increases. However, at the first two temperatures the current values have no significant changes, comparing with that before exposure to the gas. The sensitivity of the device increases from $0.37 \%$ at $T=23^{\circ} \mathrm{C}$ to $10.55 \%$ at $T=100^{\circ} \mathrm{C}$. As a result, the gas is averse to being adsorbed on GNR at temperatures under $100^{\circ} \mathrm{C}$. Also, Figure 6 shows that at $100^{\circ} \mathrm{C}$ the drain current becomes more important. This result shows that the GNR Fermi level shifts to the conduction band edges. This is due to the fact that $\mathrm{NH}_{3}$ donates electron and leaves electrons on the GNRFET channel. Thus, we can conclude that the $\mathrm{NH}_{3}$ adsorption at this temperature affects the metal/GNR junction transfer characteristics and so the device resistance.

\section{Conclusion}

In conclusion, we replicated the GNRFET-based gas sensor using the analytic model. The dependencies of $I_{d}-V_{d}$ as well as the sensing parameters and the top gate length were considered to evaluate the device performance using this model. We have shown that our device witnesses a measurable change in conductance levels once it is exposed to $\mathrm{NH}_{3}$. Two control parameters have been introduced: the parameter which governs the temperature $(\delta)$ and the parameter that monitors the gas concentration $(\lambda)$. The $I-$ $V$ curves of the gas sensors are taken into consideration to compare the transfer characteristics before and after being

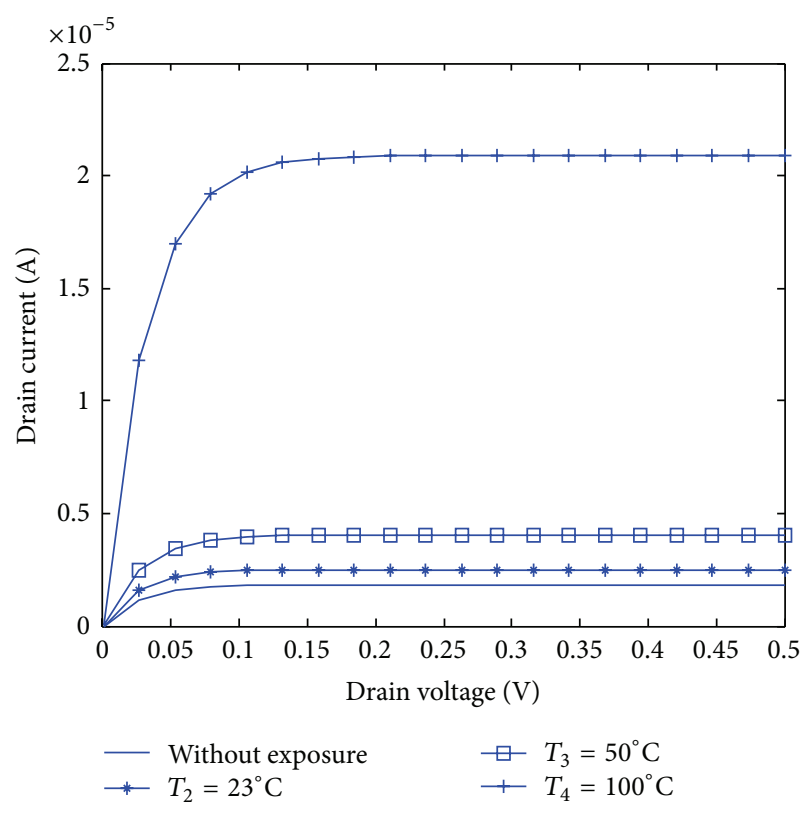

FIGURE 6: GNR-based gas sensor $I-V$ curves before and after $\mathrm{NH}_{3}$ exposure at $F=300 \mathrm{ppm}$ for $T=25^{\circ} \mathrm{C} ; 50^{\circ} \mathrm{C}$ and $100^{\circ} \mathrm{C}$ presenting higher conductivity values in elevated temperatures.

exposed to diverse $\mathrm{NH}_{3}$ concentrations and temperatures. The GNRFET-based gas sensor shows a sensitivity of about $2.7 \%$ once exposed to $1 \mathrm{ppm}$ of $\mathrm{NH}_{3}$. This interesting result makes our device an appropriate candidate to be employed in gas detection systems. We have also investigated the effect of the channel length on the ON-current and the OFF-current. We have concluded that exposure to a gas does not dope the GNRFET sensor because the doping effect would imply a shift in the "OFF" range of the transistor. Thus the gas molecule adsorption modifies the Fermi level alignment between the graphene nanoribbon and the metal in the contact region.

\section{Competing Interests}

The authors declare that they have no competing interests.

\section{References}

[1] S. Das, I. Lahiri, C. Kang, and W. Choi, "Engineering carbon nanomaterials for future applications: energy and bio-sensor," in Micro- and Nanotechnology Sensors, Systems, and Applications III, vol. 8031 of Proceedings of SPIE, Orlando, Fla, USA, April 2011.

[2] N. Kiga, Y. Takei, A. Inaba, H. Takahashi, K. Matsumoto, and I. Shimoyama, "CNT-FET gas sensor using a functionalized ionic liquid as gate," in Proceedings of the 2012 IEEE 25th International Conference on Micro Electro Mechanical Systems (MEMS '12), pp. 796-799, Paris, France, February 2012.

[3] G. Jiménez-Cadena, J. Riu, and F. X. Rius, "Gas sensors based on nanostructured materials," Analyst, vol. 132, no. 11, pp. $1083-$ 1099, 2007.

[4] A. Misra, "Carbon nanotubes and graphene-based chemical sensors," Current Science, vol. 107, no. 3, pp. 419-429, 2014. 
[5] A. K. Geim, "Graphene: status and prospects," Science, vol. 324, no. 5934, pp. 1530-1534, 2009.

[6] L. Yang, C.-H. Park, Y.-W. Son, M. L. Cohen, and S. G. Louie, "Quasiparticle energies and band gaps in graphene nanoribbons," Physical Review Letters, vol. 99, no. 18, Article ID 186801, 2007.

[7] Y. W. Son, M. L. Cohen, and S. G. Louie, "Energy gaps in graphene nanoribbons," Physical Review Letters, vol. 97, no. 21, Article ID 216803, 2006.

[8] I. S. Amiri, A. Afroozeh, and M. Bahadoran, "Simulation and analysis of multisoliton generation using a PANDA ring resonator system," Chinese Physics Letters, vol. 28, no. 10, Article ID 104205, 2011.

[9] M. Y. Han, B. Özyilmaz, Y. Zhang, and P. Kim, "Energy bandgap engineering of graphene nanoribbons," Physical Review Letters, vol. 98, no. 20, Article ID 206805, 2007.

[10] V. Barone, O. Hod, and G. E. Scuseria, "Electronic structure and stability of semiconducting graphene nanoribbons," Nano Letters, vol. 6, no. 12, pp. 2748-2754, 2006.

[11] Z. Chen, Y.-M. Lin, M. J. Rooks, and P. Avouris, "Graphene nano-ribbon electronics," Physica E, vol. 40, no. 2, pp. 228-232, 2007.

[12] K. R. Amin and A. Bid, "Graphene as a sensor," Current Science, vol. 107, no. 3, pp. 430-436, 2014.

[13] A. H. Castro Neto, F. Guinea, N. M. R. Peres, K. S. Novoselov, and A. K. Geim, "The electronic properties of graphene," Reviews of Modern Physics, vol. 81, no. 1, pp. 109-162, 2009.

[14] E. Akbari, Z. Buntat, M. H. Ahmad et al., "Analytical calculation of sensing parameters on carbon nanotube based gas sensors," Sensors, vol. 14, no. 3, pp. 5502-5515, 2014.

[15] F. Schedin, A. K. Geim, S. V. Morozov et al., "Detection of individual gas molecules adsorbed on graphene," Nature Materials, vol. 6, no. 9, pp. 652-655, 2007.

[16] E. Akbari, Z. Buntat, A. Enzevaee, M. K. Yazdi, M. Bahadoran, and A. Nikoukar, "Sensing and identification of carbon monoxide using carbon films fabricated by methane arc discharge decomposition technique," Nanoscale Research Letters, vol. 9, no. 1, article 402, pp. 1-7, 2014.

[17] W. S. Hwang, P. Zhao, K. Tahy et al., "Graphene nanoribbon field-effect transistors on wafer-scale epitaxial graphene on $\mathrm{SiC}$ substrates," APL Materials, vol. 3, no. 1, Article ID 011101, 2015.

[18] W. S. Hwang, K. Tahy, X. Li et al., "Transport properties of graphene nanoribbon transistors on chemical-vapordeposition grown wafer-scale graphene," Applied Physics Letters, vol. 100, no. 20, Article ID 203107, 2012.

[19] P. Zhao, R. M. Feenstra, G. Gu, and D. Jena, "SymFET: a proposed symmetric graphene tunneling field-effect transistor," IEEE Transactions on Electron Devices, vol. 60, no. 3, pp. 951-957, 2013.

[20] M. J. Kiani, M. T. Ahmadi, E. Akbari, H. Karimi, and F. K. Che Harun, "Graphene nanoribbon based gas sensor," Key Engineering Materials, vol. 553, pp. 7-11, 2013.

[21] T. S. Cho, K.-J. Lee, J. Kong, and A. P. Chandrakasan, “The design of a low power carbon nanotube chemical sensor system," in Proceedings of the 45th Design Automation Conference (DAC '08), pp. 84-89, Anaheim, Calif, USA, June 2008.

[22] V. Ryzhii, M. Ryzhii, A. Satou, and T. Otsuji, "Current-voltage characteristics of a graphene-nanoribbon field-effect transistor," Journal of Applied Physics, vol. 103, no. 9, Article ID 094510, 2008.
[23] A. A. Sukhanov and Y. Y. Tkach, "Equations for the surface potential distribution in two-dimensional layer systems," Soviet Physics. Semiconductors, vol. 18, no. 7, pp. 797-798, 1984.

[24] V. I. Ryzhii and I. I. Khmyrova, "Current crowding effect in hot-electron heterostructure transistors," Soviet Physics: Semiconductors, vol. 22, p. 807, 1988.

[25] M. T. Ahmadi, Z. Johari, D. C. Y. Chek, N. A. Amin, and R. Ismail, "Modelling of graphene nanoribbon Fermi energy," Journal of Nanomaterials, vol. 2010, Article ID 909347, 6 pages, 2010.

[26] V. Kažukauskas, V. Kalendra, C. W. Bumby, B. M. Ludbrook, and A. B. Kaiser, "Electrical conductivity of carbon nanotubes and polystyrene composites," Physica Status Solidi C, vol. 5, no. 9, pp. 3172-3174, 2008.

[27] D. Gunlycke, D. A. Areshkin, and C. T. White, "Semiconducting graphene nanostrips with edge disorder," Applied Physics Letters, vol. 90, no. 14, Article ID 142104, pp. 1-3, 2007.

[28] M. Khaledian, R. Ismail, M. Saeidmanesh, and P. Khaledian, "Analytical modeling of the sensing parameters for graphene nanoscroll-based gas sensors," RSC Advances, vol. 5, no. 67, pp. 54700-54709, 2015.

[29] A. Singh, M. A. Uddin, T. Sudarshan, and G. Koley, “Tunable reverse-biased graphene/silicon heterojunction Schottky diode sensor," Small, vol. 10, no. 8, pp. 1555-1565, 2014.

[30] P. Bondavalli, P. Legagneux, and D. Pribat, "Sub ppm gas sensing using a CNTFET-based sensor array fabricated using different metals as electrodes," Journal of the Korean Physical Society, vol. 54, no. 1, pp. 510-513, 2009.

[31] P. Avouris, J. Appenzeller, R. Martel, and S. J. Wind, "Carbon nanotube electronics," Proceedings of the IEEE, vol. 91, no. 11, pp. 1772-1783, 2003.

[32] A. Y. Goharrizi, M. Pourfath, M. Fathipour, and H. Kosina, "Device performance of graphene nanoribbon field-effect transistors in the presence of line-edge roughness," IEEE Transactions on Electron Devices, vol. 59, no. 12, pp. 3527-3532, 2012. 

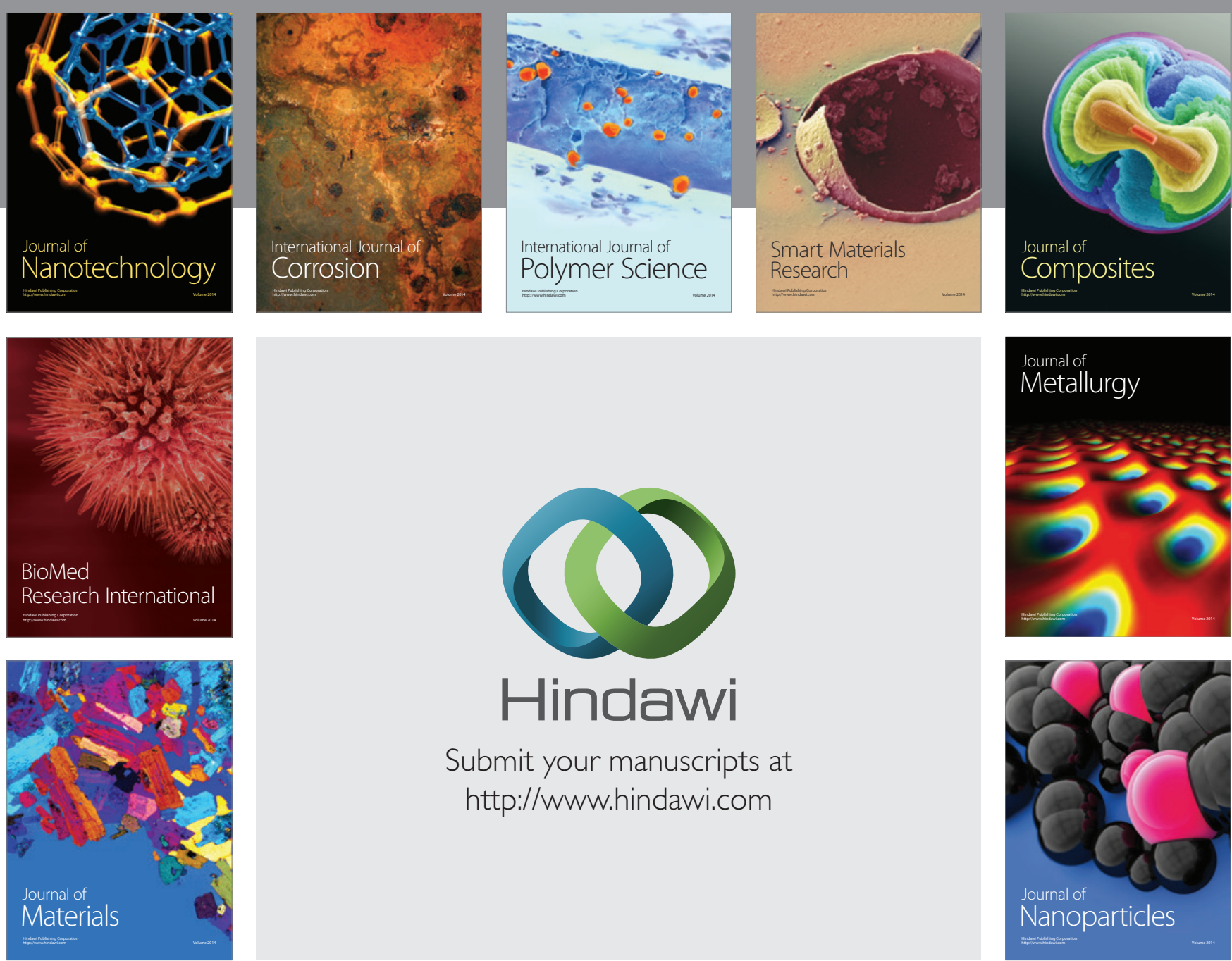

\section{Hindawi}

Submit your manuscripts at

http://www.hindawi.com

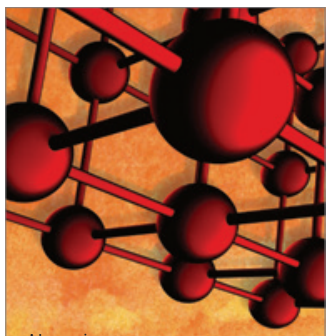

Materials Science and Engineering
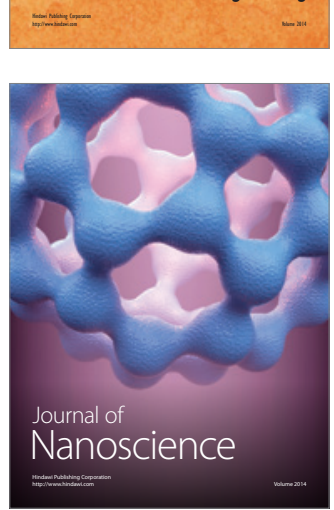
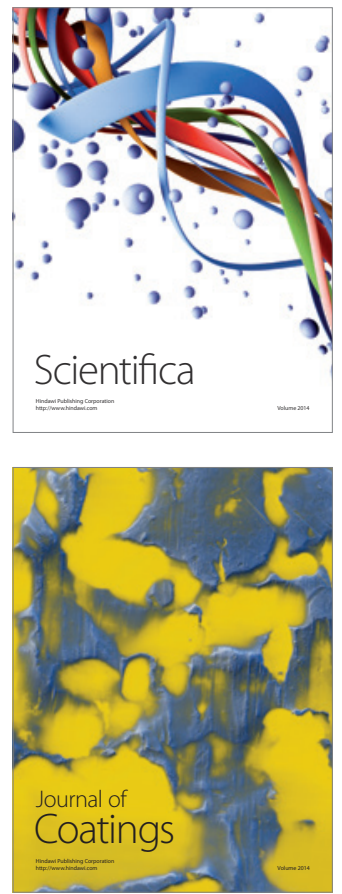
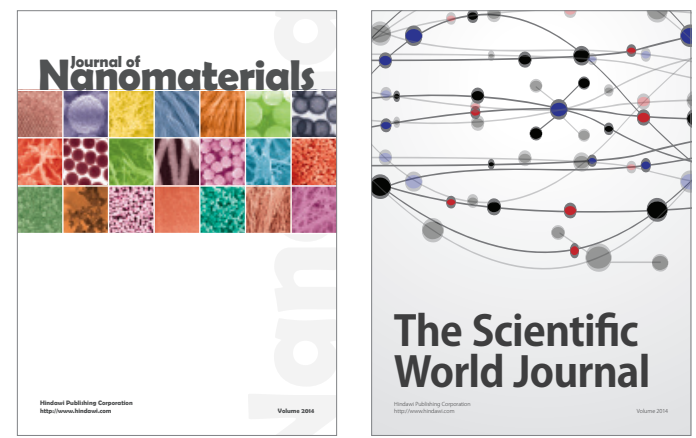

The Scientific World Journal
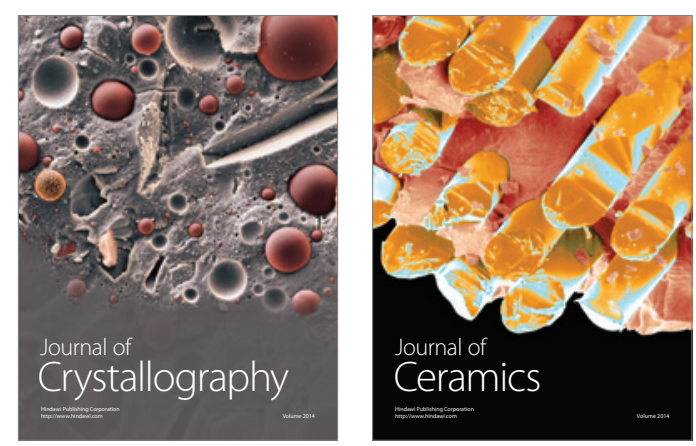
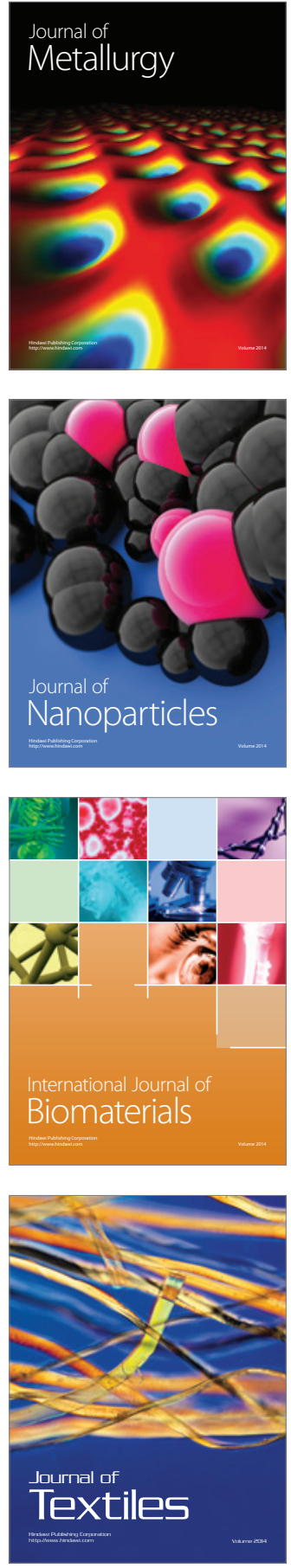